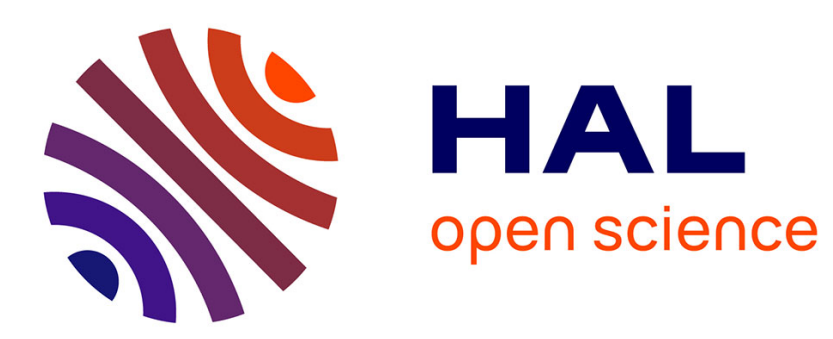

\title{
Light scattering study of the critical behaviour in a ternary microemulsion system
}

\author{
P. Honorat, D. Roux, A.M. Bellocq
}

\section{To cite this version:}

P. Honorat, D. Roux, A.M. Bellocq. Light scattering study of the critical behaviour in a ternary microemulsion system. Journal de Physique Lettres, 1984, 45 (19), pp.961-968. 10.1051/jphyslet:019840045019096100 . jpa-00232437

\section{HAL Id: jpa-00232437 https://hal.science/jpa-00232437}

Submitted on 1 Jan 1984

HAL is a multi-disciplinary open access archive for the deposit and dissemination of scientific research documents, whether they are published or not. The documents may come from teaching and research institutions in France or abroad, or from public or private research centers.
L'archive ouverte pluridisciplinaire HAL, est destinée au dépôt et à la diffusion de documents scientifiques de niveau recherche, publiés ou non, émanant des établissements d'enseignement et de recherche français ou étrangers, des laboratoires publics ou privés. 
Classification

Physics Abstracts

$64.70 \mathrm{~J}-78.35-82.70$

\title{
Light scattering study of the critical behaviour in a ternary microemulsion system
}

\author{
P. Honorat, D. Roux and A. M. Bellocq \\ Centre de Recherche Paul-Pascal, Domaine Universitaire, 33405 Talence Cedex, France \\ (Reçu le 25 juin 1984, accepté le ler août 1984)
}

\begin{abstract}
Résumé. - Le diagramme de phases composition-température du système AOT-eau-décane présente une ligne de points critiques. Nous avons examiné le comportement critique de ce système en fonction de deux paramètres qui contrôlent les interactions entre gouttelettes $\mathbf{E} / \mathrm{H}$, à savoir la température et le rapport eau sur savon. Le comportement critique est étudié à l'aide de la diffusion de la lumière. Nos résultats indiquent un comportement de type liquide-gaz. Ils sont en bon accord avec les théories du groupe de renormalisation pour les phénomènes critiques.
\end{abstract}

\begin{abstract}
The phase diagram composition-temperature of the AOT-water-decane system presents a line of critical points. This line has been approached along two paths which control the interactions between $\mathrm{W} / \mathrm{O}$ droplets. The two parameters investigated are the temperature and the water over surfactant ratio. The critical behaviour has been followed by using light scattering. The data indicate a liquid-gas type behaviour and are in good agreement with the renormalization group theories for critical phenomena.
\end{abstract}

\section{Introduction.}

Over the last few years a large number of papers have been devoted to the experimental study of critical phenomena in mixtures involving surfactants. Two-, three-, four-, and even fivecomponent systems were investigated [1-14]. The current experimental interest mainly arises from the intricate results obtained so far. For some systems, experimental data are in good agreement with those predicted by the renormalization group theories while for other ones, there is evidence for a more complex behaviour. In some cases, the transition is discussed in terms of a liquid-gas transition due to interactions between micelles. Indeed both experimental and theoretical [15-18] results have pointed out the importance of the interactions between water in oil droplets on the stability of oil rich microemulsions. Up to now, most of the papers have dealt with quaternary mixtures made of water-oil-surfactant-alcohol. It has been shown that when interactions become strongly attractive, there is evidence for a critical behaviour from light scattering $[8,11]$. This critical-like behaviour is far from being fully understood. A better knowledge of this phenomenon requires further studies on simpler systems. This prompts us to investigate a ternary system whose structure is described as a dispersion of water in oil droplets in interaction. The system chosen is made of AOT-water-decane.

Previous studies by light and neutron scattering have clearly indicated the existence of water droplets dispersed in a continuous oil phase for the water-oil-AOT systems [19-26]. Furthermore these results have shown unambiguously that the size of the water droplets increases with the 
ratio of water to AOT in the system. Within the past few years a critical behaviour has been observed by light and neutron scattering in the AOT-water-decane system as temperature is varied [5-7]. Besides it has been found that in this system an increase in attraction between droplets is achieved either by raising temperature [18] or by increasing the water over surfactant ratio at fixed temperature [26].

Our interest in this paper has been in the study by light scattering of the critical behaviour along these two paths. This study allows us to confirm the existence of an apparent field variable recently evidenced in quaternary mixtures [10]. This field variable is the water over surfactant ratio.

Because the phase equilibria of surfactant mixtures are generally very complicated, the knowledge of the evolution of the phase boundaries with composition and temperature is a fundamental step toward the understanding of the physical properties of these systems. Therefore, before discussing our light scattering results, we present in the first part the phase diagram of the system studied.

\section{Phase diagram.}

The phase diagram has been constructed according to a procedure previously described [16]. The surfactant AOT was supplied by FLUKA and the decane was a $99 \%$ label product from FLUKA. Purification of AOT was made according to the procedure described by H. Kunieda and K. Shinoda [27].

In some conditions, we have observed that AOT undergoes hydrolysis and forms the ethyl hexyl alcohol and sulfosuccinic acid. Gas chromatography has been used to detect the presence of alcohol and to measure its concentration. After one month at $25{ }^{\circ} \mathrm{C}$, less than $1 \%$ of the AOT surfactant was hydrolyzed. This quantity increases rapidly as the temperature is raised. The kinetics of hydrolysis has been recently investigated [28]. Therefore, all our experiments were performed within one day after sample preparation.

Figure 1 shows the phase diagram of the AOT- $\mathrm{H}_{2} \mathrm{O}$-decane system at $25^{\circ} \mathrm{C}$. This diagram was first established by Assih et al. [28]. At $25^{\circ} \mathrm{C}$, three one-phase regions are observed. The region richest in oil and AOT (region $\mathrm{L}_{2}$ ) is the inverted micellar domain. The mixtures observed in region $L_{1}$ are isotropic whereas those located in region $L C$ are birefringent. The essential feature

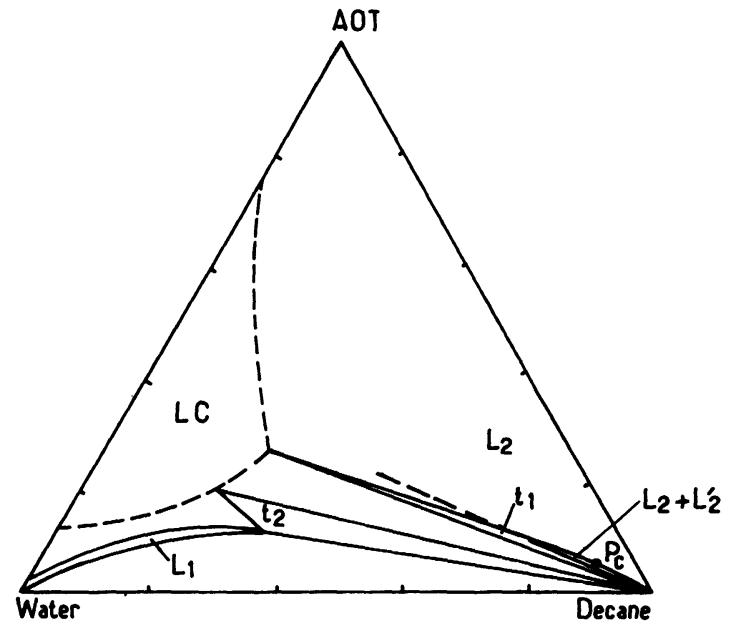

Fig. 1. - Phase diagram (expressed in weight) of the system AOT-water-decane at $25^{\circ} \mathrm{C}$. The dashed limits have not been determined precisely. 
of this diagram is the occurrence of a critical point on the demixing curve of the inverted microemulsion domain $\left(\mathbf{P}_{c}\right)$. The direct consequence of this critical point is the observation of a narrow two-phase region $\left(\mathrm{L}_{2}-\mathrm{L}_{2}^{\prime}\right)$ where two micellar phases are in equilibrium. Firstly, the critical composition has been determined by examination of the relative volumes of the phases which separate in the very close vicinity of the coexistence curve. The point where the volumes are equal is the closest to the critical point. The critical composition has also been determined by measurements of the intensity of light scattered by the mixtures located along the coexistence curve. The three one-phase domains observed $\mathrm{L}_{2}, \mathrm{~L}_{1}$ and $\mathrm{LC}$ are separated by two three-phase regions $\mathrm{L}_{2} \mathrm{~L}_{2}^{\prime}$ $L C\left(t_{1}\right)$ and $L_{2}^{\prime} L C L_{1}\left(t_{2}\right)$. As temperature is varied, a line of lower critical points is generated in the diagram composition-temperature. The composition of the critical point changes very little when the critical temperature varies. By decreasing the temperature, the extent of the $L_{2}-L_{2}^{\prime}$ region decreases. At $20^{\circ} \mathrm{C}$, both regions $L_{2}-L_{2}^{\prime}$ and $t_{1}$ have disappeared. The line of critical points terminates in a lower critical end point. The critical temperature and concentration may change from batch to batch because of the effect of purity or degradation of AOT. At temperature below $20^{\circ} \mathrm{C}$, the oil rich microemulsion domain is bounded by a two-phase region where the microemulsion phase is in equilibrium with the mesomorphic LC phase.

\section{Results and discussion.}

The determination of the phase diagram of the AOT-water-decane system at several temperatures has shown the existence of a line of lower critical points. Previously the critical behaviour of this system has been investigated at one fixed composition by light and neutron scattering [5-7]. It has been found that the correlation length $\xi$ measured by dynamic light scattering and the forward intensity $I(0)$ diverge at the critical temperature $T_{\mathrm{c}}=36.01{ }^{\circ} \mathrm{C}$ according to the power laws $\xi=\xi_{0} \varepsilon^{-\gamma}$ and $I(0)=I_{0} \varepsilon^{-\gamma}$ where $\varepsilon=\left(T_{\mathrm{c}}-T\right) / T_{\mathrm{c}}, v=0.75, \gamma=1.22$ and $\xi_{0}=12.2 \AA$. The values of $v$ and $\xi_{0}$ determined by neutron scattering [6] are in good agreement with those measured by light [5]. The value of $\gamma$ determined by neutron $(\gamma=1.61 \pm 0.09)$ is significantly larger than that measured by light.

As mentioned above, we have investigated the critical behaviour of the AOT-water-decane along two different paths which correspond to an increase of the attractions. The first one is identical to that previously followed [5-7]. Along this first path, the critical point is approached by raising the temperature at fixed composition. In the second approach, the temperature is held constant and the water over surfactant ratio is varied. In both approaches to the critical point, static and dynamic light scattering experiments were performed in the single phase domain.

3.1 CRITICAL Behaviour AT FiXed COMPOSITION. - In order to examine whether the critical exponents were the same at different points of the line of critical points, we have performed light scattering experiments at two critical temperatures different from those previously reported in references [5] and [6]. The compositions of the two samples studied are given in table I. The experimental set-up has been described elsewhere [8]. The angular dissymmetry of the total scattered intensity was obtained by measurements at five angles ranging from $40^{\circ}$ to $140^{\circ}$. The samples were held in sealed cylindrical glass cells, temperature was controlled within $0.01{ }^{\circ} \mathrm{C}$. The scattered intensity was found to obey the Ornstein-Zernike relation which is expected to be valid in the critical region

$$
I(q)=\frac{I(0)}{1+\xi^{2} q^{2}}
$$

where $q$ is the scattering vector and $\xi$ the correlation length of the concentration fluctuations. Both $\xi$ and $I(0)$, which is proportional to the osmotic compressibility of the droplet microemul- 
Table I. - Compositions in weight of the two samples studied as a function of temperature and $v_{t}$ $\gamma_{t}$ and $\xi_{0}$ results.

\begin{tabular}{|l|c|c|c|c|}
\hline Sample & $(1)$ & $(2)$ & $(\mathrm{a})$ & (b) \\
\hline$T_{\mathrm{c}}\left({ }^{\circ} \mathrm{C}\right)$ & 26.30 & 30.02 & 36.01 & 43.3 \\
\hline Decane & 87.27 & 86.96 & 89.96 & 89.07 \\
Water & 7.72 & 7.56 & 6.16 & 7.07 \\
AOT & 5.01 & 5.48 & 3.88 & 3.86 \\
$v_{t}$ & $0.76 \pm 0.05$ & $0.71 \pm 0.05$ & $0.75 \pm 0.05$ & $0.72 \pm 0.04$ \\
$\gamma_{t}(\AA)$ & $1.30 \pm 0.08$ & $1.25 \pm 0.05$ & $1.22 \pm 0.05$ & $1.61 \pm 0.09$ \\
$\xi_{0}(\AA)$ & $7.2 \pm 2$ & $9.3 \pm 2$ & $12.2 \pm 2$ & $11.0 \pm 2.1$ \\
\hline
\end{tabular}

(a) Light data from Ref. [5].

(b) Neutron data from Ref. [6].

sion, diverge at the two critical temperature studied. The log-log plots of $I(0)$ and $\xi$ versus the reduced temperature $\varepsilon=\left(T_{\mathrm{c}}-T\right) / T_{\mathrm{c}}$ are shown in figure 2 . The data are well fitted to power laws :

$$
\xi=\xi_{0} \varepsilon^{-v_{t}} \quad \text { and } \quad I(0)=I_{0} \varepsilon^{-\gamma_{t}}
$$
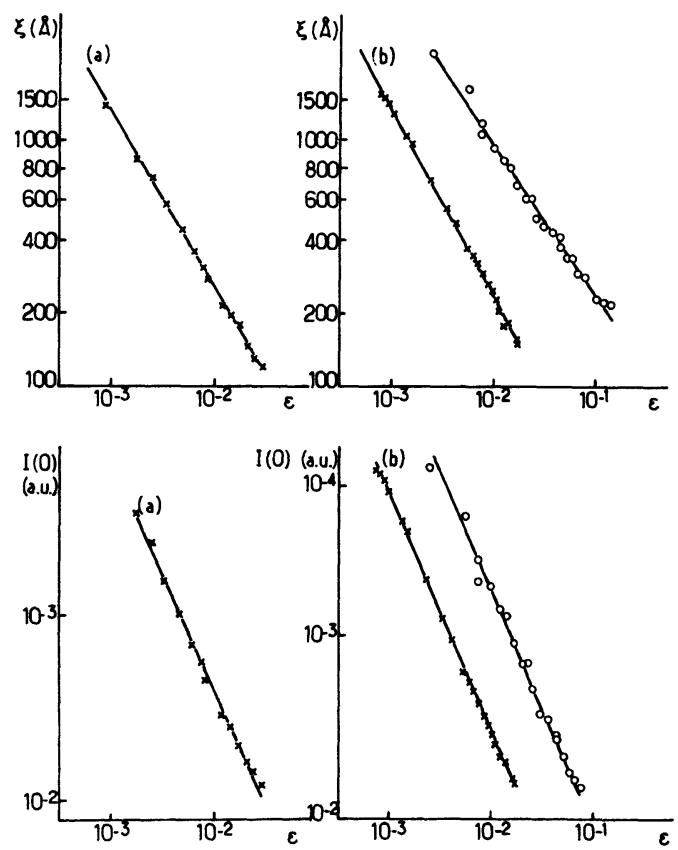

Fig. 2. - Correlation length $\xi$ and total intensity $I(0)$ (in arbitrary units) versus the reduced temperature $\varepsilon=\left(T_{\mathrm{c}}-T\right) / T_{\mathrm{c}}$ or the reduced variable $\varepsilon=\left(X_{\mathrm{c}}-X\right) / X_{\mathrm{c}}$. Data $(\times):$ the critical point is approached along an isocomposition path by raising temperature; a) sample $2, T_{\mathrm{c}}=30.02{ }^{\circ} \mathrm{C}$; b) sample 1 , $T_{\mathrm{c}}=26.30^{\circ} \mathrm{C}$. Data $(\mathrm{O}):$ the critical point is approached along an isothermal path $\left(T=25.25^{\circ} \mathrm{C}\right)$ by varying $X$. 
The $t$ index indicates that the critical point is approached in varying temperature. The values obtained by a least square fitting procedure for $T_{\mathrm{c}}$, the critical exponents $v_{t}$ and $\gamma_{t}$ and the scale factors $\xi_{0}$ are listed in table I. The $v_{t}$ and $\gamma_{t}$ values obtained at the two temperatures are very close to each other and are also very close to those found at $T_{\mathrm{c}}=36.01{ }^{\circ} \mathrm{C}$ [5]. Then in the range of critical temperatures investigated, the critical exponents $v_{t}$ and $\gamma_{t}$ exhibit a weak temperature dependence. It may be noted that our data confirm the difference, previously mentioned, between the values of $\gamma$ measured by light and neutron. We do not presently understand the origin of this difference. The large value of $\xi_{0}$ suggests that the scale of the scattered units is not at the molecular level but corresponds to an aggregate. Such large values of $\xi_{0}$ have also been reported in micellar and microemulsions systems $[3,5,12]$. The value of $\xi_{0}$ increases with temperature. Such variation likely reflects the increase of the range of attractions with temperature [18].

We have also measured on the same samples the autocorrelation function of the scattered light. In all cases, it was found a single exponential even near the critical point. From these measurements we have deduced the linewidth $\Gamma$ of the scattered light. Mode-mode coupling theory of Kawasaki [29] gives a relation between the linewidth $\Gamma$ and the scaled correlation length $x=q \xi$.

$$
\Gamma=R q^{2} k_{\mathrm{B}} T H(x) / 6 \pi \eta \xi
$$

where $\eta$ is the shear viscosity of the solution and $R$ a constant equal to 1.16 in binary mixtures [30] and which varies from 1.05 to 1.40 in pure fluids [30].

$H(x)$ is the Kawasaki formula.

$$
H(x)=3 / 4\left(1+x^{-2}+\left(x-x^{3}\right) \arctan (x)\right) .
$$

In order to test whether our results follow this formula we have measured the viscosity $\eta$ of the microemulsion. $\eta$ increases from 1.9 to $3.5 \mathrm{cp}$ as temperature varies between 20 and $30^{\circ} \mathrm{C}$. The reduced linewidth $\Gamma^{*}=\Gamma 6 \pi \eta / k_{\mathrm{B}} T q^{2}$ versus $q \xi$ is plotted in figure 3 . The full line is the universal function $R H(q \xi) / q \xi$, where $\xi$ is the correlation length measured from static data. A value of $R=1.2$ gives a very good agreement between experiments and theory. This value is close to that previously reported [5].

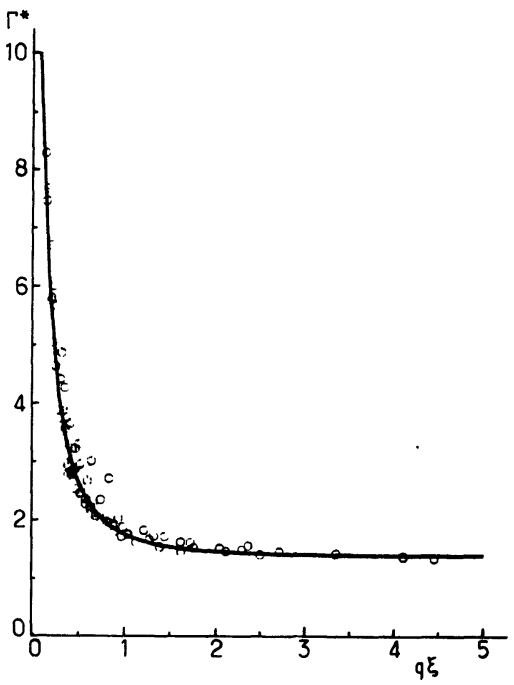

Fig. 3. - Scaled linewidth $\Gamma^{*}$ as a function of $q \xi$ for different temperatures (sample $2, T_{\mathrm{c}}=30.02{ }^{\circ} \mathrm{C}$ ). $\xi$ is the correlation length measured in static experiments. The solid line is the Kawasaki curve for $R=1.2$. 
3.2 CRitical behaviour at FiXed temperature. - We have tried to approach the critical point by varying the water over surfactant ratio at fixed volume fraction $\left(\phi_{\mathrm{H}_{2} \mathrm{O}}+\phi_{\mathrm{AOT}}=12.7 \%\right)$ and fixed temperature $T=24.5^{\circ} \mathrm{C}$. As the water over surfactant ratio (denoted by $X$ in the following and expressed in weight) is larger than 1.595 a narrow two-phase region where two isotropic microemulsions are in equilibrium is observed. It extends from $X=1.595$ to $X=1.662$. The shape of the coexistence curve in the vicinity of the critical point has been determined by composition analysis of the phases in equilibrium. The data show that the water over surfactant ratio in the lower phase is equal to the value $X$ of the overall mixture. The conservation of the $X$ ratio in the phases in equilibrium even far from the critical point is evidence that $X$ behaves as a field variable. This result confirms the generality of the field character of this variable in oil rich microemulsions [10]. From phase composition data, it is also found that the difference in decane concentrations $d_{1}$ and $d_{2}$ in the coexisting phases follows the power law $d_{1}-d_{2} \approx \varepsilon^{\beta_{x}}$. The index $x$ indicates that the critical point is approached in varying $x, \varepsilon$ represents the distance from the critical point and is defined as $\varepsilon=\left(X_{\mathrm{c}}-X\right) / X_{\mathrm{c}}, X_{\mathrm{c}}$ is the value of $X$ at the critical point $\left(X_{\mathrm{c}}=1.595\right)$. The critical exponent $\beta_{x}$ is found to be $0.4 \pm 0.05$.

We have also performed light scattering experiments in the single phase domain as a function of $X$ along the path just above defined $\left(\phi_{\mathrm{H}_{2} \mathrm{O}}+\phi_{\mathrm{AOT}}=12.7 \% ; T=25.25^{\circ} \mathrm{C}\right)$. All the quantities measured, the correlation length $\xi$, the intensity $I(0)$ and the linewidth of the scattered light $\Gamma$ show large variation as $X$ is varied from 1.376 to 1.563 ; this latter value is that of the critical point. In figure 2 are given in $\log$ - $\log$ plots the variations of $\xi$ and $I(0)$ versus $\varepsilon$ the reduced value of $X$. The data are well fitted to the power laws

$$
\xi=\xi_{0} \varepsilon^{-v_{x}} \quad \text { and } \quad I(0)=I_{0} \varepsilon^{-\gamma_{x}}
$$

with $v_{x}=0.61 \pm 0.06$ and $\gamma_{x}=1.26 \pm 0.10$.

The $v_{x}$ and $\gamma_{x}$ values are in good agreement with the Ising values. This result is in accordance with the theoretical predictions of Griffiths and Wheeler [31] for ternary mixtures. In these systems, the Ising exponents are expected to be obtained as one imposes the condition of a constant field and a constant density. This condition corresponds to path II, where the critical point is approached by varying the field $X$ at a fixed field (temperature) and a constant density (decane

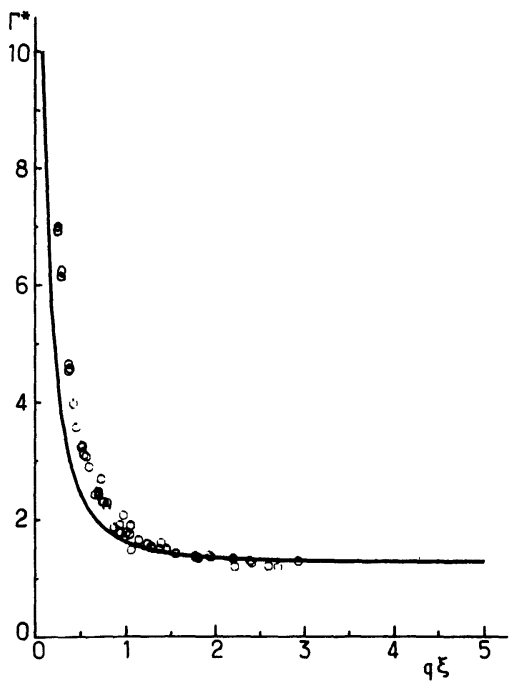

Fig. 4. - Scaled linewidth $\Gamma^{*}$ as a function of $q \xi$ for different values of $X\left(T=25.25^{\circ} \mathrm{C}\right)$. The solid line is the Kawasaki curve for $R=1.1$. 
concentration). In the first path the critical point is approached at constant overall concentration as a function of temperature. In this case where one has a double density constraint (decane and $X$ concentrations). The exponents are expected to be renormalized by the $1 /(1-\alpha)$ factor (Fisher renormalization) [32]. The slightly higher value of $v_{1}$ found along the first path could be in agreement with this renormalization $(v /(1-\alpha)=0.72$ with $v=0.63$ and $\alpha=0.1)$. Such renormalized value has been found near an ordinary plait point of a ternary liquid mixture [33].

The reduced linewidths $\Gamma^{*}$ measured in the same set of samples are plotted versus $q \xi$ in order to be compared to the Kawasaki equation. In these samples viscosity increases from 2.6 to $3.3 \mathrm{cp}$ as $X$ varies from 1.376 to 1.56 . Actually the experimental data are well fitted by the theoretical curve in the vicinity of the critical point with $R=1.1$ (Fig. 4). The systematic excess discrepancy noticed far from $X_{\mathrm{c}}$ probably indicates the existence of a non-critical background contribution to the dynamics of the concentration fluctuations. Indeed the size of the particles continuously varies with $X$. The change in size is larger along the $X$ scan than what has been measured as temperature is changed [18]. Indeed the water core radius increases approximatively from 50 to $60 \AA$ as the $X$ ratio increases from 1.376 to 1.563 [25].

\section{Conclusion.}

In pure fluids, interactions are essentially a function of temperature. In microemulsion systems several experimental studies have shown that interactions between $\mathrm{W} / \mathrm{O}$ droplets are also dependent on many others parameters such as the micellar size or the chemical nature of the components. Therefore, in these systems, the phase transition may be controlled not only by temperature but also by any parameter which affects the interactions. Huang had shown recently that the carbon number of oil can be used as a variable to approach the critical point [18]. In this Letter we have shown that the droplet size which is directly related to the water over surfactant ratio $X$ is also a " critical " variable. Our results identify a parallel between the two variables temperature and $X$ that is consistent with the fact that phase separation is due to interactions.

The critical phenomenon of the ternary microemulsion system AOT-water-decane, as measured by the divergences of the correlation length and the scattered intensity is well described by power laws. Depending upon the path of approach to the critical point ( $X$ scan or temperature), the exponents $v$ and $\gamma$ take the Ising or the renormalized Ising values.

\section{References}

[1] Lang, J. C., Morgan, R. D., J. Chem. Phys. 73 (1980) 5849.

[2] Corti, M., Degiorgio, V., Phys. Rev. Lett. 45 (1980) 1045 ; J. Phys. Chem. 85 (1981) 1442.

[3] Corti, M., Minero, C., Degiorgio, V., J. Phys. Chem. 88 (1984) 309.

[4] Appell, J., Porte, G., J. Physique Lett. 44 (1983) L-689.

[5] Huang, J. S., Kim, M. W., Phys. Rev. Lett. 47 (1981) 1462.

Kim, M. W., Huang, J. S., Phys. Rev. B 26 (1982) 2703.

[6] Kotlarchyx, M., Chen, S. H., Huang, J. S., Phys. Rev. A 28 (1983) 508.

[7] Toprakcioglu, C., Dore, J. C., Robinson, B. H., Howe, A., Chieux, P., J. Chem. Soc. Faraday Trans I 80 (1984) 413.

[8] Fourche, G., Belloce, A. M., Brunetti, S., J. Colloid Interface Sci. 89 (1982) 427.

[9] Belloce, A. M., Bourbon, D., Fourche, G., Lemanceau, B., J. Colloid Interface Sci. 89 (1982) 427.

[10] Roux, D., Belloce, A. M., Phys. Rev. Lett. 52 (1984) 1895.

[11] Cazabat, A. M., Langevin, D., Meunier, J., Pouchelon, A., Adv. Coll. Int. Sci. 16 (1982) 175 ; J. Physique Lett. 43 (1982) L-89.

[12] Abillon, O., Chatenay, D., Langevin, D., Meunier, J., J. Phys. Lett. 45 (1984) L-223.

[13] Dorshow, R., De Buzzaccarini, F., Bunton, C. A., Nicoli, D. F., Phys. Rev. Lett. 47 (1981) 1336.

[14] Tabony, J., Drifford, M., De Geyer, A., Chem. Phys. Lett.96 (1983) 119. 
[15] Roux, D., Belloce, A. M., Leblanc, M. S., Chem. Phys. Lett. 94 (1983) 156.

[16] Roux, D., Belloce, A. M., Physics of Amphiphiles : Micelles, Vesicles and Microemulsions, ed. by V. Degiorgio and M. Corti (North Holland, Amsterdam) 1984.

[17] Safran, S. A., Turkevich, L. A., Phys. Rev. Lett. 50 (1983) 1930.

[18] Kotlarchy,, M., Chen, S. H., Huang, J. S., Kim, M. W., Phys. Rev. A 29 (1984) 2054.

[19] Zulauf, M., Eicke, H. F., J. Phys. Chem. 83 (1979) 480.

[20] Day, R. A., Robinson, B. H., Clarke, J. H. R., Doherty, J. V., J. Chem. Soc. Faraday Trans I 75 (1979) 132.

[21] Robinson, B. H., Toprakcioglu, C., Dore, J. C., Chieux, P., J. Chem. Soc. Faraday Trans I80 (1984) 13.

[22] Cabos, C., Delord, P., J. Appl. Cryst. 12 (1979) 502; J. Phys. Lett. 41 (1980) 455.

[23] Sein, E., Lalanne, J. R., J. Colloid Interface Sci. 72 no 2 (1979) 363.

[24] Gulari, E., Bedwell, B., AlkafajI, A., J. Colloid Interface Sci. 77 (1980) 202.

[25] Kotlarchy,, M., Chen, S. H., Huang, J. S., J. Phys. Chem. 86 (1982) 3273.

[26] Honorat, P., Thesis Bordeaux 1984.

[27] Kunieda, H., Shinoda, K., J. Colloid Interface Sci. 70 (1979) 577.

[28] Delord, P., Larche, F. C., J. Colloid Interface Sci. 98 (1984) 277.

Assih, T., Delord, P., LaRChe, F. C., Symposium on Surfactants in Solution, Lund, K. L. Mittal Ed. (Plenum Press) 1984, p. 1821.

[29] Kawasaki, K., Phys. Lett. A 30 (1969) 325 ; Phys. Rev. A 1 (1970) 1750.

[30] Beysens, D., NATO Adv. Study Inst. Ser., 82, 72 (1982) 25.

[31] Griffiths, R. B., Wheeler, J. C., Phys. Rev. A 2 (1970) 1047.

[32] Fisher, M. E., Phys. Rev. 176 (1968) 257.

[33] Ohbayashi, K., Chu, B., J. Chem. Phys. 68 (1979) 5066. 